\title{
THE CARTILAGE OF THE INTRAPULMONARY BRONCHI IN NORMAL LUNGS, IN BRON- CHIECTASIS, AND IN MASSIVE COLLAPSE
}

\author{
BY \\ JOHN HAYWARD AND LYNNE McA. REID* \\ From the Royal Melbourne Hospital, Melbourne, Australia
}

(RECEIVED FOR PUBLICATION SEPTEMBER 24, 1951)

The descriptions of the lung during the latter half of the last century, notably by Aeby (1880) and Ewart (1889), and also the more modern work by Miller (1947) and Engel (1947) give descriptions of the cartilages in the trachea, carina, and main bronchi, but are disappointing in their lack of detailed information about the cartilages in the intrapulmonary bronchi. On this subject the authors have concerned themselves with the determination of the size of bronchi at which cartilage disappears. Some have also attempted an estimate of the distance of this point from the hilum. Miller states that he found cartilage disappeared from the bronchial tree when the tubes were on an average between 0.6 and $0.7 \mathrm{~mm}$. diameter. His summary of the results of other workers gave figures which ranged between $0.4 \mathrm{~mm}$. (Frankenhäuser) and $1 \mathrm{~mm}$. (Krause and others).

In a previous paper (page 89) we have shown the limited significance of measurements of bronchial diameter, especially as indications of position in the bronchial tree, and one aim of this paper is the reassessment of the point of disappearance of cartilage in terms of the number of bronchial generations from the hilum. Dissections were also made to show the gradation in the amount of cartilage along the wall of a bronchus and the shape and arrangement of cartilage at points of bronchial division. Horner (1839) and King (1840) described "saddle-shaped cartilages" and Miller (1947) has described also a circular type which he found in the lungs of man. The present paper also records the results of our examination of the bronchial cartilages in bronchiectasis. Indeed it was here that our investigations began, for it was while studying specimens of this disease, particularly those associated with massive collapse, that our interest in the bronchial cartilages was aroused and we realized that their normal anatomy had been incompletely described.

Finally, some aspects of the significance of the bronchial cartilages in pulmonary pathology will be discussed.

\section{Methods of INVESTIGATION}

Both lungs from 12 cadavers preserved by the injection of formalin into the body in the Anatomy School at the University of Melbourne were examined and found only suitable for the study of the cartilages in the proximal bronchi. Pathological changes

${ }^{*}$ Research Fellow, National Health and Medical Research Council. 
such as bronchopneumonia and tuberculosis interfered in some cases with the determination of the most distal point at which cartilage was present, and for this 12 other healthy lungs from necropsies at the Royal Melbourne Hospital were used. At first fresh specimens, specimens treated with Ranvier's alcohol as described by Miller (1947), and specimens fixed for one week after intrabronchial injection with $8 \%$ formol-saline were dissected. Soon it was found that demonstration of the cartilages was easy after formalin fixation, and subsequently this method was used exclusively.

The lungs dissected were from adults, and no attempt was made to correlate age or sex with the anatomical findings. Cartilages from large lungs were larger and thicker, but their distribution and arrangement seemed similar.

In the anatomical study four segments in each lung were selected for examination : the apical segment of the upper lobe, the apical and posterior basal segments of the lower lobe, and the lower branch in the medial segment of the middle lobe or the inferior segment of the lingula according to the side. In each segment " axial " bronchi were specially studied because, as shown by our previous work (Reid, 1950 ; Hayward and Reid, p. 89), these bronchial pathways, running the longest course to the most distal lobules and giving off the greatest number of side branches, are the most suitable for making comparisons between different segments in the same lung and the same segment in different lungs. The cartilages along selected "lateral" bronchial pathways were also noted.

Each bronchus examined was exposed by dissection and opened along its length because it was found easier to see and feel the cartilages from the inside. It also proved a simple matter to dissect off the layers internal to the cartilages so as to render them more obvious. They were examined and drawings made of them in situ with the bronchial wall spread out flat. Many, especially those at the orifices of branches, were then dissected free for closer inspection. Even the smallest plates occurring just before the final disappearance of cartilage could be seen with the naked eye in normal lungs, so that for the purposes of this investigation histological examination could be dispensed with.

The cartilages in bronchiectatic bronchi in upward of 100 lobes removed at operation were similarly examined. A few of these lobes were from children. In many of them serial sections previously prepared for another purpose were also available for study so that where pathological changes made detection of the cartilage difficult histological evidence could be obtained.

\section{Results}

Cartilage Arrangement in the Walls of Normal Intrapulmonary BRONCHI.-In the main bronchi the cartilages are, as in the trachea, in the form of C-shaped rings, sometimes joined to adjacent rings but never extending across the posterior gap which constitutes the membranous part of the tube. As they enter the lung the membranous part ends, and it is with this position that this paper begins. It is not a sharply defined region, because lung tissue bulges medially behind and in front of the main bronchi and the line of reflection of visceral on to parietal pleura at the hilum is somewhat variable.

Cartilage Arrangement Where the Bronchi Enter the Lung.-This is different on the two sides. On the right side the main bronchus gives off the upper lobe branch from its supero-lateral side and its $\mathrm{C}$-shaped rings join with the cartilages which surround the upper lobe orifice. The upper lobe bronchus immediately enters the lung, but the main bronchus continues downwards just outside the lung still with C-shaped cartilages and with its membranous part intact. As soon as it reaches 
the point just above the orifices of the bronchi to the middle lobe and to the apical part of the lower lobe it, too, enters the lung and its cartilages become irregular ; they surround the orifices of both the branches, and extend to the back, thus narrowing the membranous part which is usually closed by a separate irregular plate of cartilage filling up the posterior gap.

On the left side (Fig. 2) the main bronchus ends by dividing into upper and lower lobe branches which both enter the lung immediately and are completely surrounded by cartilage.

Cartilage Arrangement Along the Intrapulmonary Bronchi.-The cartilages in the intrapulmonary bronchi are scattered in the tough fibro-elastic adventitial layer of the bronchial wall, thickenings of which connect nearby points of adjacent cartilages forming elastic intercartilaginous ligaments. Fig. 1 is a drawing of the cartilages along an axial pathway in a posterior basal segment. The bronchus is laid open longitudinally and then drawn diagrammatically, but as nearly as possible to scale, showing the cartilages and the orifices of branches.

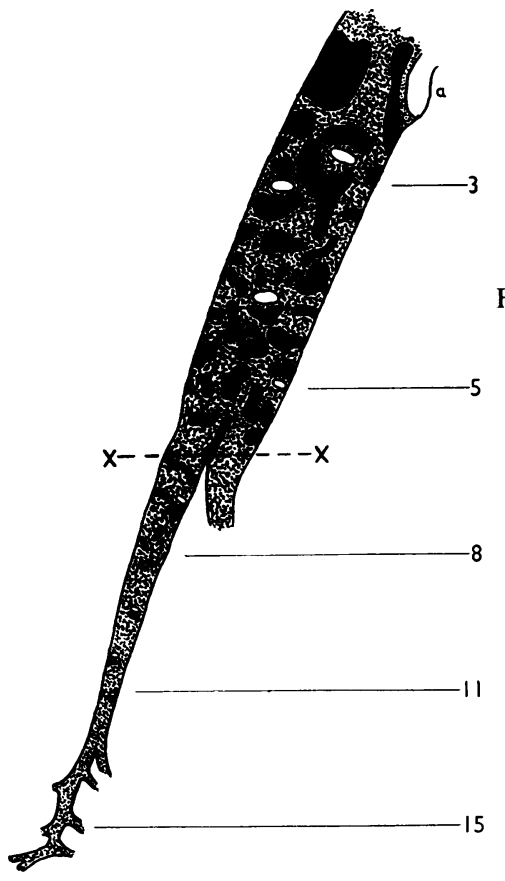

Fig. 1.-Diagrammatic representation of the plates of cartilage (black) in the wall of an axial bronchus of the posterior basal bronchopulmonary segment of an adult. The bronchus has been laid open longitudinally and is displayed flattened. $a$ is the carina between the posterior and middle basal bronchi. The openings of the side bronchi appear white, and are numbered. Proximal to the point $x$ the cartilage supports the entire circumference of the bronchial wall continuously along its length, while distally it is present only as scattered plates. In this diagram the size is reduced by one-third.

The most obvious feature brought out by the diagram is the presence of large, more closely spaced cartilages proximally and tiny, widely dispersed cartilages distally. This is characteristic of all bronchial pathways. Closer inspection immediately reveals the close relation between the cartilages and the bronchial orifices. Throughout the portion of the bronchial tree provided with cartilage no orifice is without its cartilage. Proximally cartilages surround each orifice fairly completely ; distally there is but a single tiny rod of cartilage along the distal margin of each orifice. There is, moreover, a considerable amount of cartilage in the bronchial 


\section{THE CARTILAGE OF THE' INTRAPULMONARY BRONCHI 101.}

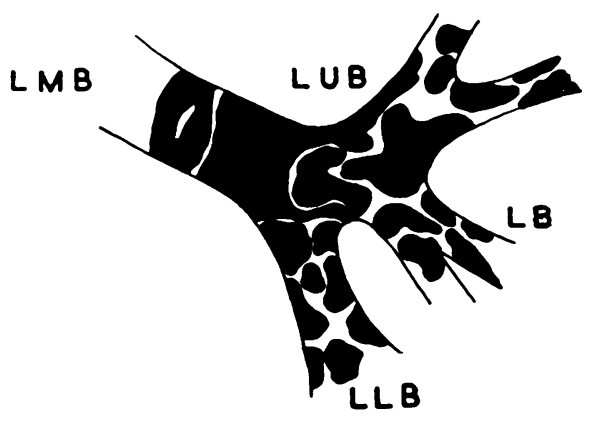

ANTERIOR VIEW



POSTERIOR VIEW

FIG. 2.-The arrangement of the cartilage (black) at the termination of the left main bronchus (L.M.B.) and around the origin of the bronchi to the left upper lobe (L.U.B.), with its lingular branch (L.B.), and its apical branch (L.A.L.) and lower lobe (L.L.B.). In the wall of the main bronchus are open rings of cartilage separated posteriorly by the membranous portion. As the bronchus branches, the rings are replaced by plates of cartilage distributed around the entire circumference of the wall. The membranous part of the main bronchus narrows progressively over its distal portion.

walls between orifices proximally, but bronchial tube cartilage (if it may be so called) as distinct from orifice cartilage is almost entirely absent distally.

Another point to be noticed is that in the proximal part the long axis of each piece of cartilage shows no constant alignment in the bronchial wall. Some cartilages have their long axis lying transversely like the rings in the trachea and main bronchi, but many of these send out processes in the direction of the tube and in others the long axis is oblique or longitudinal. All the cartilages are attached to nearby points of their proximal, distal, and transverse neighbours by ligaments, so that the cartilages and intercartilaginous ligaments in the proximal intrapulmonary bronchi together form a supporting mesh or trellis which gives longitudinal as well as circumferential stability to the tubes.

An idea of the amount of circumferential support afforded by the cartilages is best obtained after the bronchus is dissected free but before it is opened. Palpation then reveals that for a distance into the segment of about five divisions the tubes feel relatively rigid and resist occlusion by external pressure. Distal to this point they are readily compressed. The transition is moderately abrupt.

The cartilaginous intrapulmonary bronchi may therefore be divided into two parts : a proximal part receiving circumferential support from numerous cartilages scattered along the wall of the tubes on all sides as well as at the orifices, and a distal part with few or no cartilages except at the orifices which thus receive no such support. We have called the proximal part the part with circumferential cartilage. In Fig. 1 the line $\mathrm{X}-\mathrm{X}$ indicates the level of the transition between the two parts.

It will be seen that cross-sections of the proximal part would show cut surfaces of cartilage scattered round the tube on all sides and usually occupying a total of 
not less than half its circumference. Cross-sections of the distal part would show no cartilage except when taken near an orifice, and then only on one side of the tube.

The above description applies to axial pathways in all segments. Lateral bronchial pathways, which arise from axial bronchi and pass for shorter distances with fewer subdivisions to supply the more proximal lobules of segments, have no circumferential cartilage except round their orifices of origin from axial bronchi. Thereafter they resemble the distal parts of axial bronchi.

Individual Cartilages at Bronchial Orifices.-A series of cartilages round the orifices of the lobar bronchi were dissected. Those round the branches of the left main bronchus are shown in Fig. 2.

We have previously shown that bronchial divisions within the lobes and segments can be divided into two types which we have called acute angle divisions and wide angle divisions (page 94). In the former the orifices of the two divisions are separated by a sharp carina. In the latter the orifice of the branch is a hole in the side wall of the parent stem. The cartilages are somewhat differently arranged at these two types of orifice.

In orifices with a carina cartilage is always present in the carina. At this type of division in proximal bronchi (Fig. $3 a, c$; Fig. $4 a, c$ ) the carinal cartilage is large and usually extends for a short distance proximally into the parent stem at each end of the carina and distally into the adjacent walls forming the acute angle between the divisions. As a result of these extensions its shape frequently resembles a saddle. It is also joined by ligaments to other plates of cartilage surrounding the two orifices which the carina separates, thus giving them circumferential support.

The orifices of wide angle branches which appear as side holes in the parent stem are surrounded by plates of cartilage situated in the wall of the parent stem (Fig. $3 b$, $d$; Fig. $4 b, d$ ). Occasionally the cartilage round the orifice is in one continuous ring. More often it is formed of two or three plates in close apposition and joined by ligaments. In the larger branches of this type processes extending at right angles from the plates into the beginning of the wall of the side branch are usually present. They serve to maintain its wide angle with the parent stem. The most characteristic example of this type of branch is the bronchus to the apical segment of the lower lobe.

Extent of Cartilage into the Bronchial Tree.-We describe distances into the bronchial tree in terms of the number of bronchial "generations" along the bronchial pathways from the hilum. The generations of the bronchi are the segments of the tubes between successive subdivisions or branches. In all counts of bronchial generations recorded in this paper the segmental bronchi are counted a's the first generation. Each of the two bronchi produced by the first division of a segmental bronchus is regarded as belonging to the second generation. The four bronchi produced by the division of each of the two bronchi of the second generation belong to the third generation, and so on.

Axial bronchial pathways in five representative bronchopulmonary segments were traced, counting the number of bronchial generations to the level where the cartilage ceased to give circumferential support and then further to the most distal 
FIG.3.-Examples of the arrangement of cartilage around the origins of lobar and segmental bronchi. $a$ is a posterior basal segmental bronchus, $b$ and $d$ are apical bronchi of the lower lobes, $c$ is a middle lobe bronchus. They are viewed as they lie in the wall of the main bronchus, which in the case of $b$, $c$, and $d$ means the observer is looking directly into the lumen, and, in the case of $a$, across it.

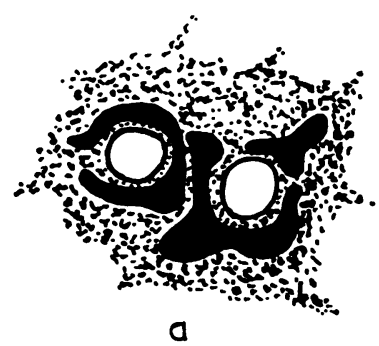

a

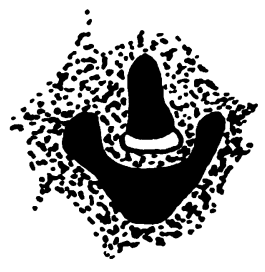

C

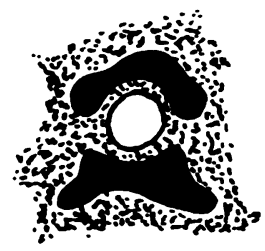

b

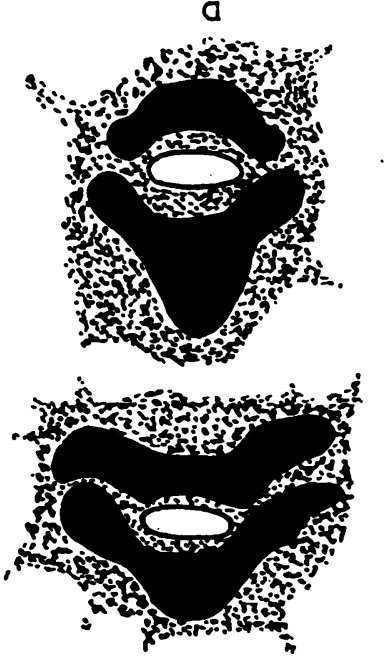

C
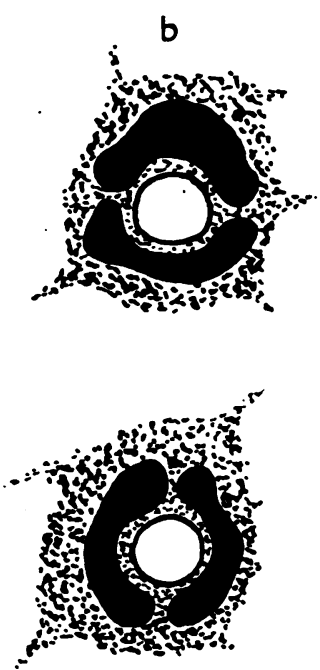

d
FIG. 4.-Examples of the arrangement of cartilages at the origins of intra-segmental branches. The cartilages are drawn as if in one plane, though they are, of course, irregular and in three dimensions. $a, c$, and $e$ are acute angle branches; $b$ and $d$ are wide angle branches. $a$ shows the orifices of the bronchi to the superior and inferior lingular segments seen by looking down a lingular bronchus. Each orifice is completely surrounded by cartilage, and the carina between them is formed by the articulation of two pieces of cartilage. In the fresh specimen this was sharp, but to show the shape of the cartilages they have been drawn flat in the diagram. $b$ and $d$ are drawn looking straight into wide angle branches, which are surrounded by pieces of cartilage. $c$ shows the orifice of a proximal acute angle division as seen obliquely from the parent stem. It shows the extensions of the carinal cartilage up into the parent stem and the separate cartilage in the parent stem which completes the circle of support round the orifice. $e$ shows a similar view of the orifice of a distal acute angle branch with only one small piece of cartilage in the carina. 
piece of bronchial cartilage. The average results of the counts are shown in the table.

TABLE

The Distribution of Cartilage in the Walls of Axial Bronchi

\begin{tabular}{c|c|c|c|c|c}
\hline Segment of Lung & $\begin{array}{c}\text { Apical } \\
\text { Upper } \\
\text { Lobe }\end{array}$ & $\begin{array}{c}\text { Medial } \\
\text { Middle } \\
\text { Lobe }\end{array}$ & $\begin{array}{c}\text { Lower } \\
\text { Lingula }\end{array}$ & $\begin{array}{c}\text { Posterior } \\
\text { Basal } \\
\text { Lower } \\
\text { Lobe }\end{array}$ & $\begin{array}{c}\text { Apical } \\
\text { Lower } \\
\text { Lobe }\end{array}$ \\
\hline $\begin{array}{c}\text { Generations of bronchi with circum- } \\
\text { ferential cartilage } \ldots\end{array}$ & 4 & 4 & 5 & 6 & 4 \\
$\begin{array}{c}\text { Total generations of bronchi in which } \\
\text { cartilage identifiable } \\
\text {.. .. }\end{array}$ & 9 & 10 & 11 & 10 & 8 \\
\hline
\end{tabular}

The slight differences between segments seem to be related to the differences in their maximum length from apex to periphery, the lingula being the longest and the apical segment of the lower lobe the shortest. The longer a bronchial pathway is the more divisions there are likely to be along its length and the more of its proximal divisions have cartilage in their walls. However, the differences are slight, and for practical purposes it is sufficiently accurate to state that cartilage extends along all axial pathways for at least 10 generations and in about the first five of these it is circumferential.

It happens that axial pathways usually consist of between 20 and 25 generations to the terminal bronchioles. Hence without being too precise it may be stated that the first half of the generations of an axial pathway of the bronchial tree have cartilage and in the first quarter the cartilage is circumferential.

Lateral pathways have circumferential cartilage only at their orifice. Beyond this there are only occasional scattered plates of cartilage in the wall and small pieces at the points of division, usually for about five or six generations.

We made no actual measurements of the diameter of the bronchi at the level of the most distal pieces of cartilage, but it seemed that their internal diameter would approximate to the figures in the literature quoted at the beginning of this paper.

Distribution of CaRTILAGe IN BRONCHIECTASIS.-Reid (1950) has shown that in cases of bronchiectasis there is a varying reduction in the number of bronchial subdivisions which can be correlated with the different morphological types of bronchial dilatation. The distribution of cartilage in these types was considered.

In cylindrical and moniliform bronchiectasis the bronchial part of the bronchial tree is intact and the distribution of cartilage appears normal (Fig. 6).

In saccular or cystic bronchiectasis there is considerable bronchial destruction, and in this type the disappearance of cartilage is greatest. Usually only three to five generations of bronchi remain in the bronchopulmonary segments. The proximal of these generations are not appreciably dilated and their cartilage appears normal. They lead into the large saccules whose walls contain no cartilage. Distal to the saccules there is no evidence of cartilage (Fig. 5).

In the intermediate group of cases of bronchiectasis there is a wide range of morphological appearances with a corresponding variation in the maximum number of patent bronchial generations, but the tubes are never patent beyond the limit to 


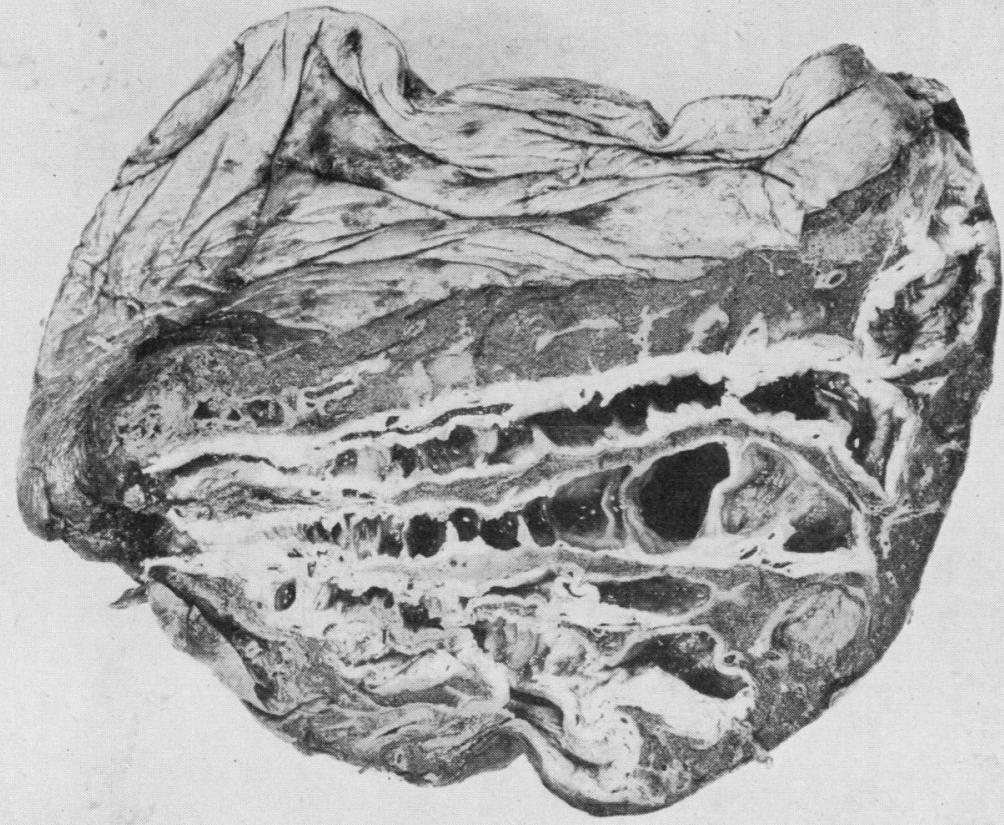

Fig. 5.-A middle lobe showing saccular bronchiectasis. The subpleural sacs have no cartilage in their walls. Only three generations of patent bronchi can be counted within the segment.

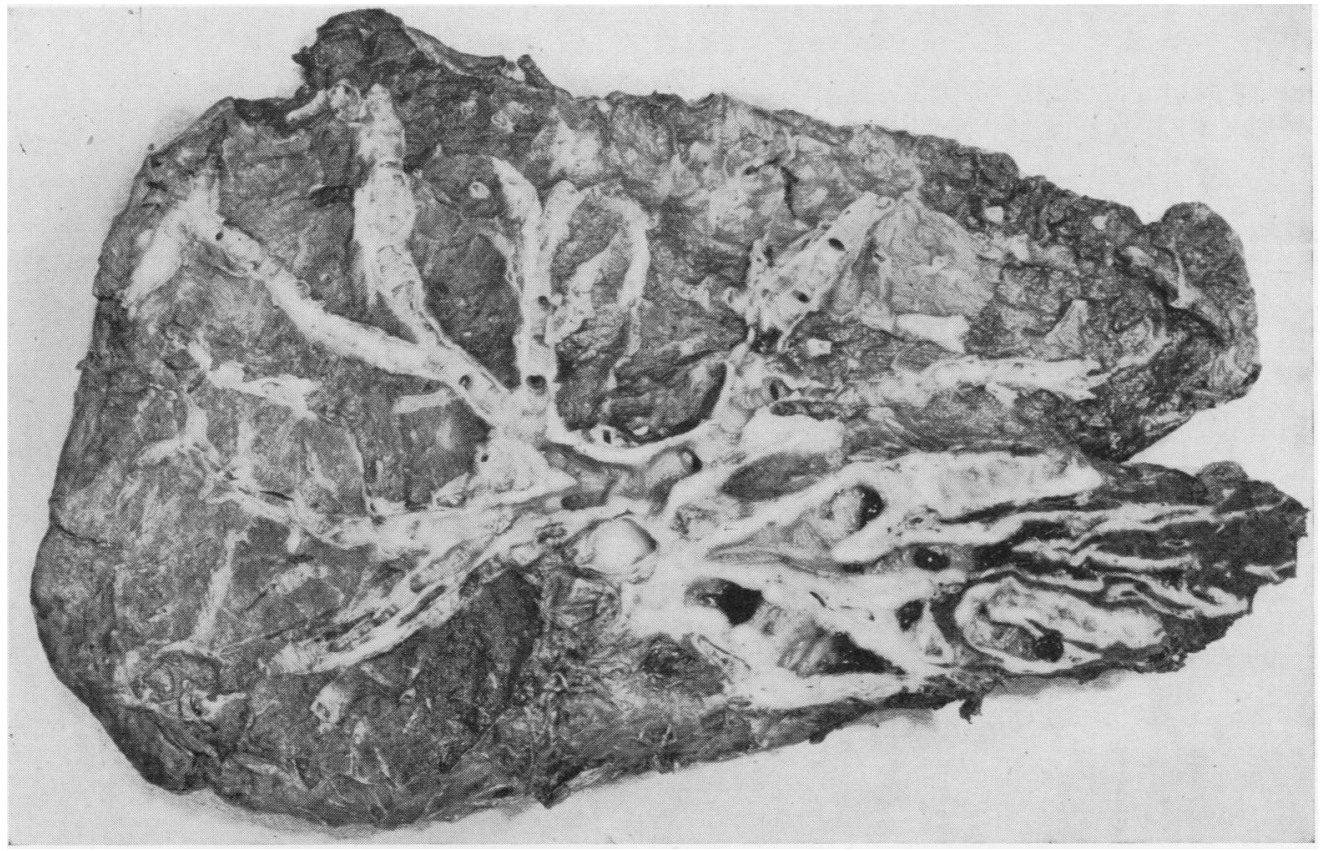

FIG. 6.- - Left lung removed for bronchiectasis. The lower lobe (in lower right corner of photograph) is massively collapsed and the dilated bronchi extend for a maximum of seven generations to the point of fibrous obliteration, beyond which they continue as fibrous cords, two of which are visible at the base. The upper lobe is aerated and its bronchi show localized dilatations which had the appearance of moniliform bronchiectasis in the bronchogram. 
which cartilage normally extends, and the average number of patent generations is six. In this group the lumen of the bronchus is obliterated by fibrous tissue, and when the point of obliteration is proximal to the normal point of disappearance of cartilage the continuation of the tubes is frequently represented by a strand of fibrous tissue in which remnants of cartilage may persist (Fig. 6). Along the patent tubes, which in these cases are dilated to a variable degree, cartilage, save in two conditions, is present in apparently normal distribution. When there are saccules either at the end of the patent bronchi (Fig. 5) or along their course, and when there are points of relative narrowing in which the whole thickness of the bronchial wall is replaced by scar tissue (Fig. 7), cartilage is absent.

In many cases of this intermediate group there is chronic massive collapse of the lobes, as they are much reduced in volume and quite solid with close crowding of the bronchi (Fig. 6, lower lobe). In this subgroup the average maximum number of bronchial generations which remain patent is five, with variation of only one or two from case to case. Lateral bronchi are obliterated immediately beyond their origin from axial bronchi. Both in specimens and in bronchograms they appear as small lateral culs-de-sac along the dilated axial bronchi. This means that all the bronchial pathways in these collapsed lobes are obliterated except those which have been shown to be normally supplied with circumferential cartilage. In many cases of this type the bronchi, though irregular, are scarcely dilated at all. In these cases the circumferential cartilage is still present along the whole length of the patent tubes. However, in common with all members of the intermediate group, these cases sometimes develop saccules from which, as usual, cartilage is absent.

\section{Discussion}

As by definition bronchi contain cartilage and bronchioles do not, by describing the distribution of cartilage this paper automatically defines the bronchial part of the tree. Furthermore, these dissections show that the proximal bronchi have much more cartilage in their walls than the distal ones. Consistent with this structural difference is the behaviour of the larger bronchi, which, in many pathological states, contrasts with that of the more distal ones. The larger bronchi frequently remain open when the more distal ones have been affected like the bronchioles and alveoli and have collapsed. As many of the proximal intrapulmonary bronchi are visible bronchoscopically, this fact is repeatedly confirmed by bronchoscopists, who are familiar with the fact that the lumen of these is never obliterated save by severe local processes which damage the wall, such as inflammatory or neoplastic strictures or pressure of diseased lymph nodes.

The support afforded by the cartilage is most conclusively shown in cases of massive collapse in which the bronchial wall is normal, as for example when it is due to a tension pneumothorax or pleural effusion. In the airless lobe or lung the proximal bronchi are always patent. If the massive collapse is due to a removable obstruction, such as a plug of viscid sputum, the open proximal bronchi are seen as soon as the plug is sucked out and before the lobe re-aerates. In specimens of massively collapsed lobes obtained at operation or necropsy the patency of the proximal bronchi is confirmed, and the distal bronchi and bronchioles are found to be as collapsed as the alveoli, with nothing inside them except perhaps a little 


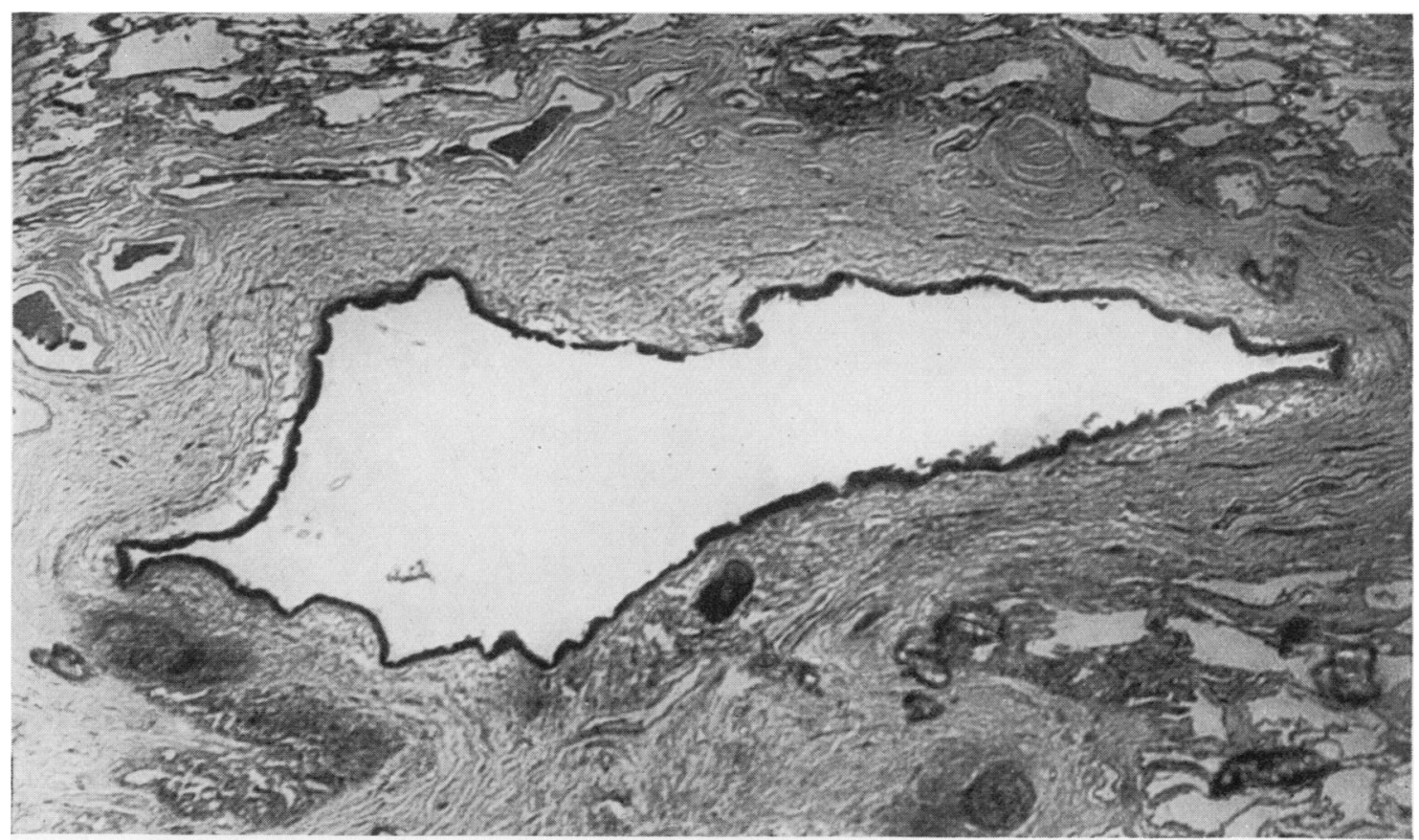

FIG. $7 a$.

Fig. 7.-(a) Photomicrograph of a stricture of a bronchus occurring in the fifth generation of an axial bronchus. The epithelium rests on dense scar tissue in which there is no evidence of muscle, glands, or cartilage. (b) Shows the bronchus distal to the stricture in which cartilage, glands, and some muscle are evident. The cartilage shows areas of bone formation.

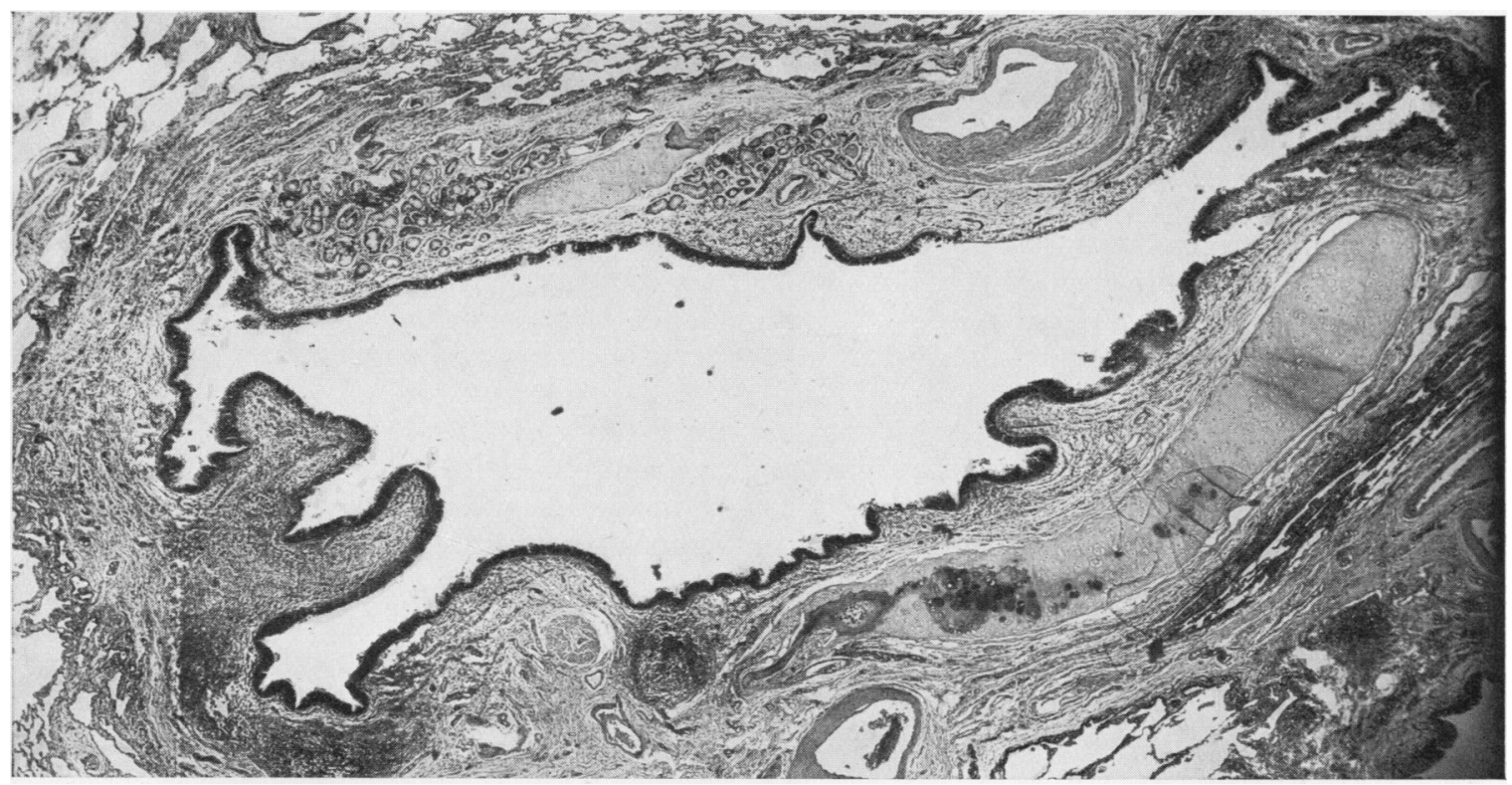

FIG. $7 b$. 
secretion. In certain circumstances the secretion may be sufficient to distend them, but they are always airless.

The proximal bronchi, as well as being patent, may also be dilated. The relation between massive collapse and certain types of bronchiectasis is well established, and there is much evidence that massive collapse without pneumothorax or pleural effusion always results in dilatation of the proximal bronchi. In our cases of bronchiectasis associated with massive collapse we found, as stated above, that the bronchi which remained patent and were dilated corresponded to those which normally have circumferential cartilage, and that the lumen of these bronchi ended abruptly at the level where cartilage normally ceases to be circumferential. It seemed probable that the distal bronchi collapsed at the time of the original massive collapse and never reopened.

When a massively collapsed lobe reaerates before irreversible inflammatory changes have occurred, the distal bronchi reopen and the proximal bronchi return to normal diameter. In the literature describing reversible bronchiectasis there are some illustrations of bronchograms done within three to eight weeks of the onset of massive collapse (e.g. Jennings, 1937 ; Lander and Davidson, 1938). The reproductions are small, but they do show that it is only the proximal several generations of axial bronchi which are outlined by lipiodol and are dilated and that the peripheral part of the bronchial tree has failed to fill. Even at this early stage, therefore, the distal bronchi are not open, but the fact that the changes may be reversed indicates that this occlusion is initially due to a cause which may be transient in operation. Examination of operation specimens of chronic massive collapse indicates that it is not due to the bronchi being blocked by secretion. In such cases excision has usually been advised because the patient presents with the clinical features of bronchiectasis. The symptoms have been chronic cough and sputum over many years and bronchography shows the expected deformity of the bronchial tree. On an average five generations is the most of an axial pathway which is demonstrated by the lipiodol. If suitable serial bronchograms are available they usually show that there has been little change over a long period.

We could find no record in the literature of a bronchogram having been done within a few hours or even days of the onset of massive collapse, doubtless for the same reason that we have not done one ourselves : the patient is too ill for the investigation to be justifiable. Definite evidence of the time of onset of the dilatation of the proximal bronchi and collapse of the distal bronchi is therefore lacking, but there seems no reason why they should not occur simultaneously with the absorption of the alveolar air as part of the process of massive collapse and not as an aftermath.

Adams, Hrdina, and Dostal (1935), reporting their investigations on the effect in dogs of stenosis of a bronchus produced by painting it with silver nitrate, comment that in the massive collapse which resulted the proximal bronchi were distended with secretion while the distal bronchi and bronchioles were collapsed. There is thus experimental as well as clinical and pathological evidence that there is a difference in response between proximal and distal bronchi in massive collapse.

All this evidence seems to justify the conclusion that the dividing line between proximal patency, perhaps with dilatation, and distal collapse of the bronchi in massive collapse is at the level where the bronchial cartilage ceases to give circum- 
ferential support, and further that it is this support which determines the difference in response between the proximal and distal bronchi. Their behaviour at the onset of the collapse seems to be due largely to simple mechanical factors. The distal tubes collapse with the alveoli because their walls lack sufficient support to withstand the deflating effect of the absorption of air as the lobe collapses. When collapse is complete and the distal bronchi, bronchioles, and alveoli are all airless they form a continuous, solid, relatively inelastic investment round the proximal bronchi, which alone remain patent because their cartilaginous support is sufficient to withstand the deflating force. The solid lung then transmits the negative intrapleural pressure directly to their walls.

The intrapleural pressure is well known to be lower in massive collapse, and its dilating effect on the still patent tubes has been shown by Lander and Davidson (1938), who found that its reduction by injecting air into the pleural cavity in cases of bronchiectasis reduced the amount of bronchial dilatation.

Lander (1936), after stating that in massive collapse with "immobile" obstruction the bronchi in which there are "rings" of cartilage do not collapse but those without this support do, goes on to say that in massive collapse with "mobile" obstruction the finer "cartilage-free bronchi" become distended and dilated as the obstructing plug of mucus is sucked out into them by the distal absorption of air. Our work shows that it is not the finer bronchi or the bronchioles but the proximal bronchi which dilate in bronchiectasis with massive collapse, and that the distal bronchi and bronchioles not only collapse at the outset, as Lander seemed to realize, but remain collapsed permanently in those cases in which there is no re-aeration of the lobe. Also, any secretion subsequently found in them can, in our opinion, be formed locally and is not part of the original obstructing plug.

The effect of the cartilage on the appearance of the bronchial tree in massive collapse has been considered in the last section. Survival of the cartilage in bronchiectasis suggests that the continuity of the bronchial wall has never been broken. In cylindrical and moniliform bronchiectasis it is likely that all the bronchial walls have always been intact because in these cases the distribution of the cartilage is normal. These cases also show that the cartilage will allow a limited amount of dilatation. In moniliform bronchiectasis the points of relative narrowing are at the bifurcations where the cartilages offer the greatest support.

Disappearance of the cartilage in bronchiectasis suggests that inflammation of a type very destructive to the tissues of the bronchial wall has occurred, and the cartilage is seldom if ever destroyed without the other special structures such as muscle and glands being destroyed also. Since cartilage is always absent from the walls of bronchiectatic saccules, it would seem that dilatations of this size cannot occur without loss of the support of the cartilages and the intercartilaginous ligaments, and that they must be the product of considerable bronchial destruction. It is likely that they frequently arise from complete necrosis of a length of bronchus and the adjacent lung tissue with subsequent epithelization of the resultant cavity. It is necessary to count the number of generations between such a saccule and the segmental bronchus to determine whether the area normally contains cartilage. Usually such saccules are within the first five generations, where cartilage is normally present.

We do not consider that the absence of cartilage must be taken as evidence of a congenital developmental defect, and we regard most of the cases of saccular 
bronchiectasis previously called congenital as acquired diseases. Indeed, we have not yet seen a case of this type in which we believed that the saccules were congenital.

\section{SUMMARY}

The normal anatomy of the bronchial cartilages is described. In particular it is shown that they extend for approximately 10 generations along axial pathways and give circumferential support to the proximal half of these generations. The arrangement of the cartilages at bronchial orifices is given special attention.

The changes in the extent of the bronchial cartilage in bronchiectasis are described. It is normal in cylindrical and moniliform bronchiectasis, suffers the greatest reduction in saccular bronchiectasis, and is reduced to a variable degree in the various intermediate forms of bronchiectasis. It is scarcely ever found in the walls of bronchiectatic saccules. In bronchiectasis associated with massive collapse only the bronchi normally supplied with circumferential cartilage remain patent.

Because of this and other evidence presented it seems that in massive collapse all the bronchi without circumferential support and all the bronchioles collapse with the alveoli, and that it is the circumferential support of the cartilage in the proximal bronchi which keeps them open and thereby exposes them to the dilating force of the negative intrapleural pressure.

\section{REFERENCES}

Adams, W. E., Hrdina, L., and Dostal, L. E. (1935). J. thorac. Surg., 4, 377.

Aeby, C. (1880). Der Bronchialbaum der Säugethiere und des Menschen. Leipzig. Quoted by Miller. Engel, S. (1947). The Child's Lung. London.

Ewart, W. (1889). The Bronchi and Pulmonary Blood-Vessels. London.

Horner, W. E. (1839). A Treatise on Special and General Anatomy. Philadelphia. Quoted by Miller. Jennings, G. H. (1937). Brit. med. J., $2,963$.

King, J. (1840). Guy's Hosp. Rep., 5, 237.

Lander, F. L. (1936). Proc. roy. Soc. Med., 29, 1383.

Lander, F. P. L., and Davidson, M. (1938). Brit. J. Radiol., 11, 65.

Miller, W. S. (1947). The Lung. Baltimore.

Reid, L. McA. (1950). Thorax, 5, 233. 